\title{
Status and Prospects for observation of top quark pair production in ATLAS
}

Borut Paul Kerševan, Ljubljana Group

Faculty of Mathematics and Physics University of Ljubljana Eksperimental Particle Phys. Dept. Jozef Stefan Institute 


\section{The LHC Operation in 2010/2011}

- High energy proton collisions at $7 \mathrm{TeV}$ are ongoing.

- Target $L_{\text {int }} \sim 1 \mathrm{fb}^{-1}$ by end of 2011 .

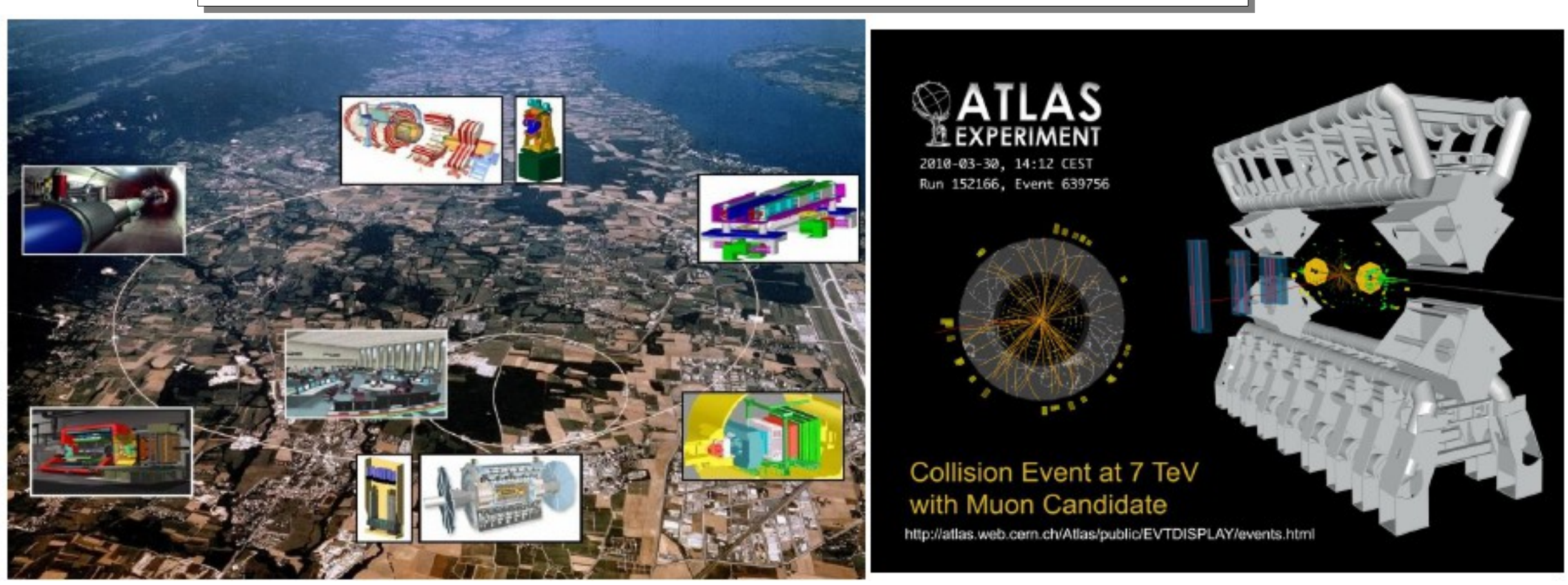

LHC program for 2010-11 focusses on:

- Detector commisioning.

- Standard Model Physics.

- Physics beyond TeV scale:

- Possible surprises (Susy, Higgs, Exotics..) 


\section{ATLAS Performance so far}

- Expect a few pb-1 by the end of summer 2010

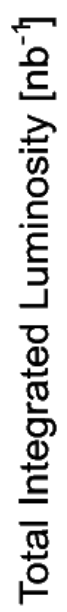
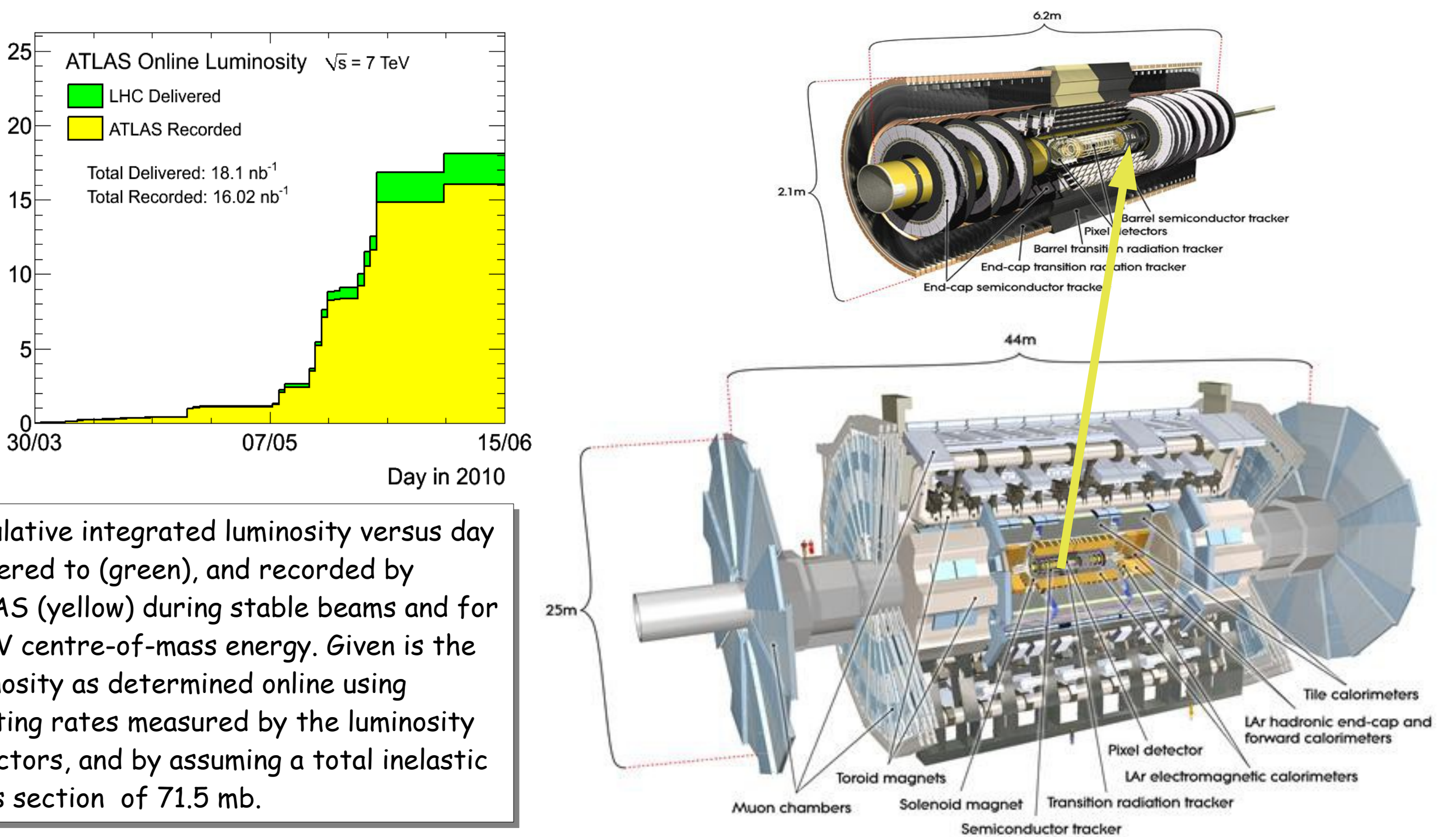

Cumulative integrated luminosity versus day delivered to (green), and recorded by ATLAS (yellow) during stable beams and for $7 \mathrm{TeV}$ centre-of-mass energy. Given is the luminosity as determined online using counting rates measured by the luminosity detectors, and by assuming a total inelastic cross section of $71.5 \mathrm{mb}$. 


\section{Roadmap to Top quark Re-discovery}

- We are heavily involved in understanding our detector and basic reconstruction objects:

- leptons, jets, b-tagging, Missing transverse energy (MET)

- understanding $Q C D$ processes

- First steps towards $Z$ and $W+$ jets observation.

- Need to understand the above items when proceeding to top quark re-discovery.
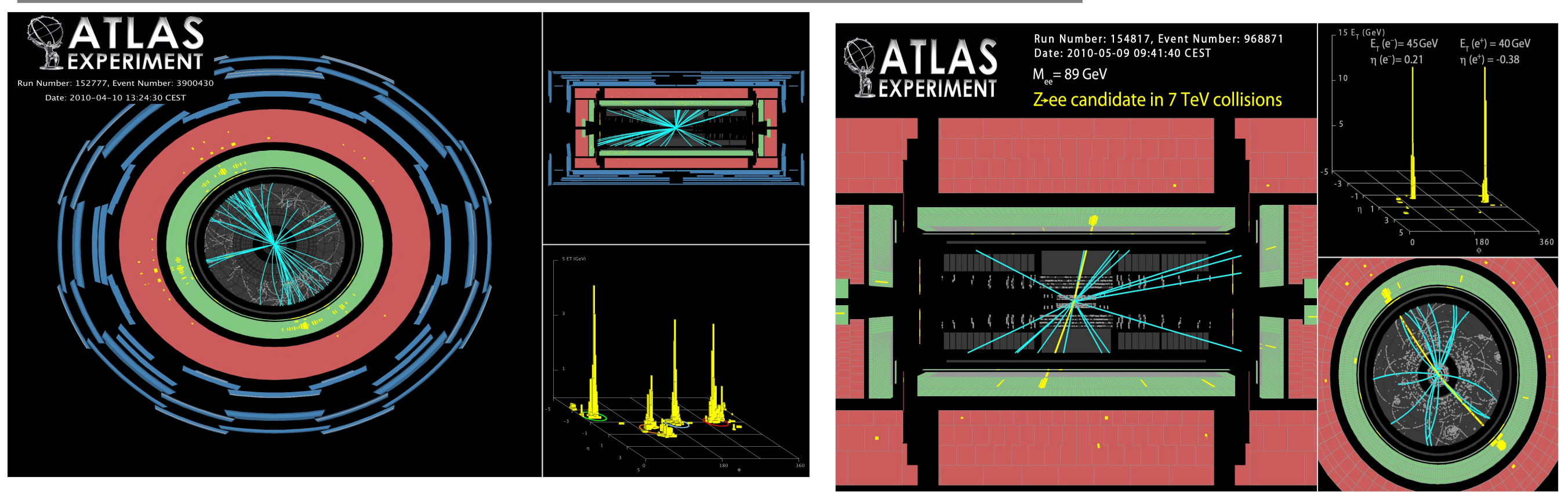


\section{Top Quark Physics @ ATLAS}

- Top physics @ ATLAS:

- First:

- Top re-discovery: top-antitop production

- Detector calibrations Jet energy scale (JES) $b$-tagging efficiency

- Next:

- Precision tests of SM

- Search beyond SM: top-antitop resonances anomalous coulplings non-SM top production
QCD: top-antitop production:

$1 \mathrm{fb}^{-1} @ 7$ TeV: $160 \mathrm{k}+t$ pairs produced top re-discovery process

$\sigma_{\mathrm{tt}}=160 \mathrm{pb} @ 7 \mathrm{TeV} \sim 20 \times$ Tevatron

$$
\text { g }
$$

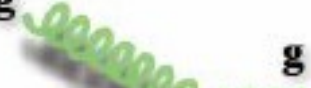

g

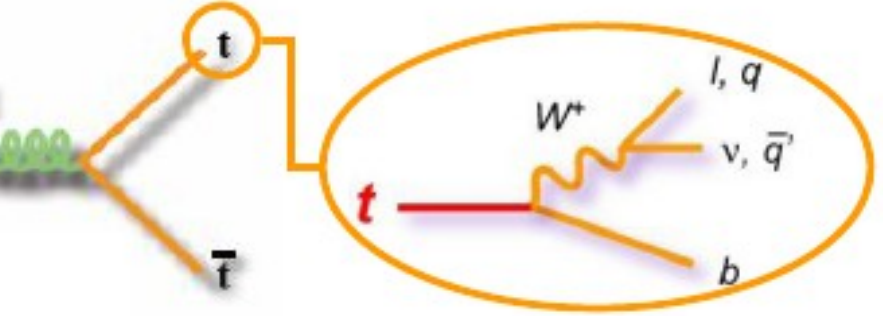

EW: single top production:

Sensitive to beyond SM physics

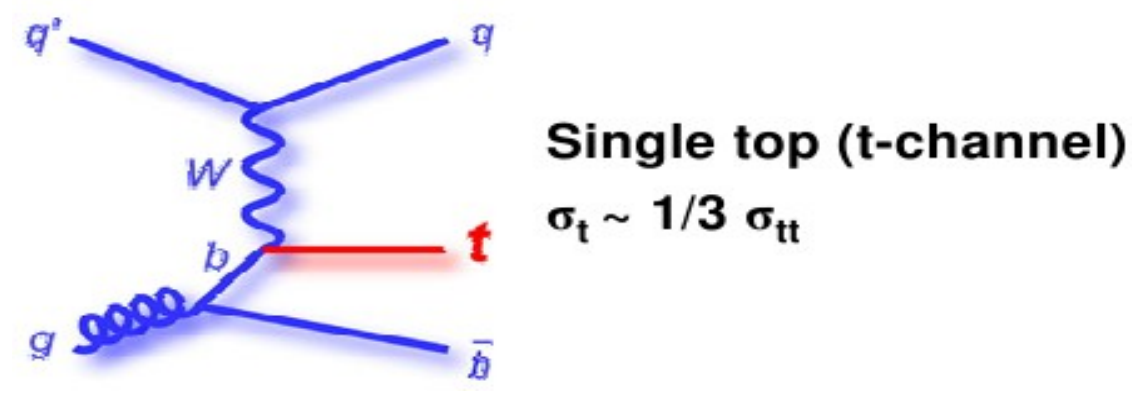




\section{Top Pair Decay Channels}

- Top-antitop decay modes:

- Di-leptonic channel:

- low statistics (9\%)

- clean signature S/B 4.5-6.5

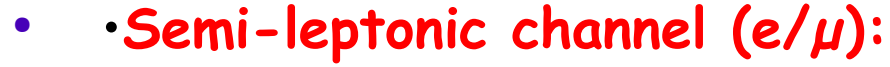

- $45 \%$ of total

- $S / B \sim 1$ (without b-tag)

- Visible top and/or W invariant mass peaks helps

- Fully hadronic channel:

- $46 \%$ of total:

- high QCD backgrounds.

- Not easy to trigger on (no leptons, need b-tag trigger?)

- Not for early data!
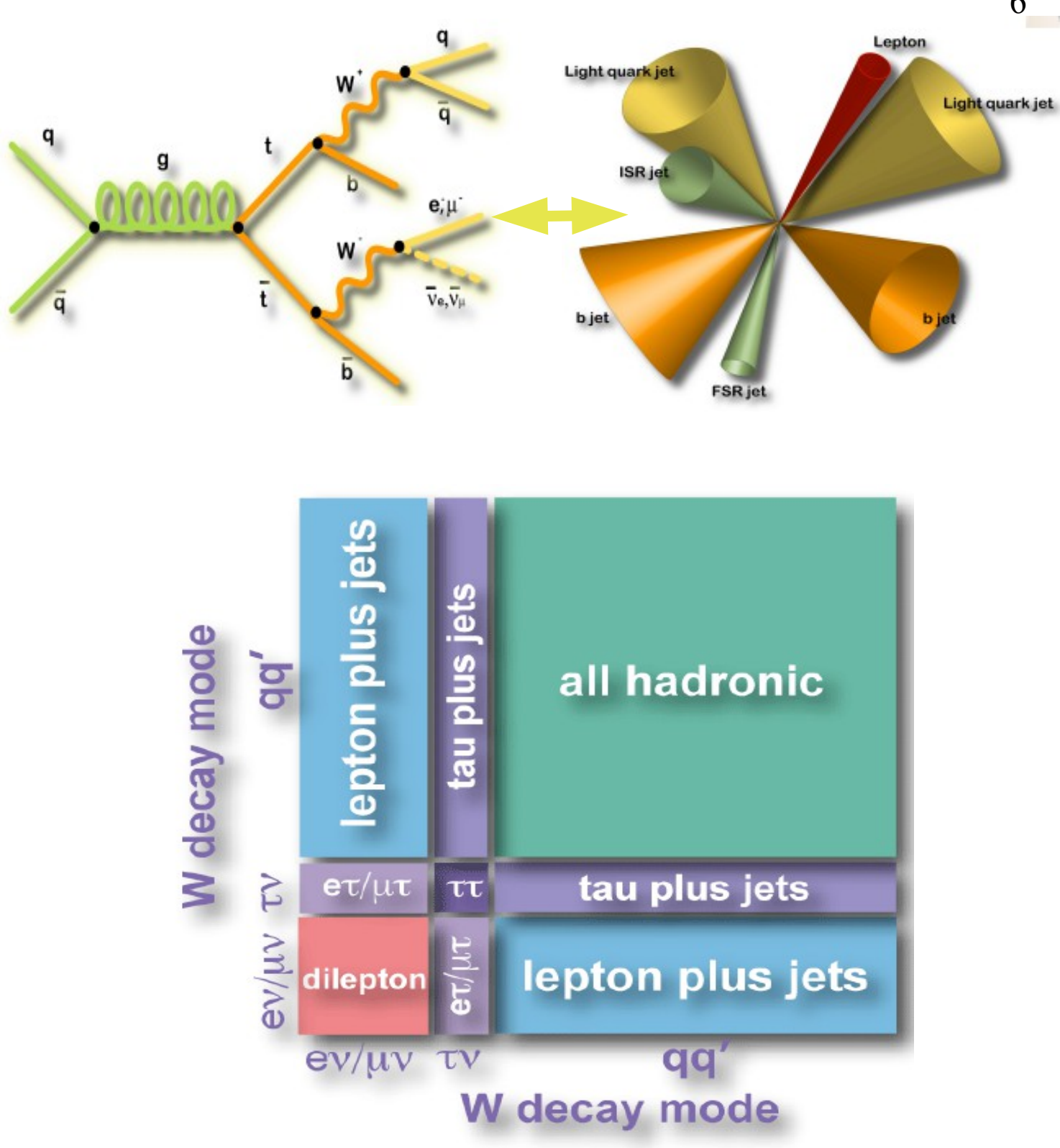


\section{First top Measurements}

The first top measurement to be done with early data is the ttbar $x$-section determination

- Dilepton channel

- 2 opp. charged leptons

- 2 jets or more

- Missing $E_{T}$

- Backgrounds:

- 2 real leptons

- Drell-Yan

$$
t \bar{t} \rightarrow \ell^{+} v \ell^{-} v b \bar{b}
$$

- Dibosons

- Single-top (Wt)

- fake leptons

- W+jets, QCD multijet $\left[>1000 \times \sigma_{t+}\right]$

- Strategy:

- Veto $Z$ window and require MET
- Lepton+jets channel

- 1 lepton

- 4 jets or more $\quad \begin{aligned} & t \bar{t} \rightarrow \ell v q q b \bar{b} \\ & \ell=e, \mu\end{aligned}$

- Missing $E_{T}$

- Backgrounds:

- combinatorial

- W+jets $\left[\sim 40 \times \sigma_{t+}\right]$

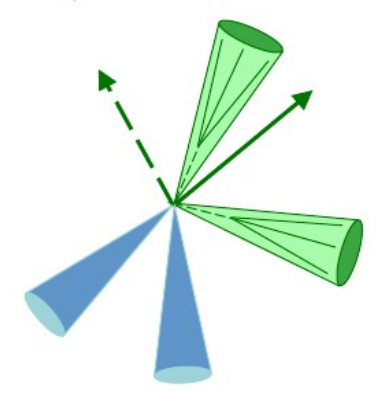

- single-top

- fake leptons from QCD multijet $\left[\gg 1000 \times \sigma_{t+}\right]$

- Strategy:

- reconstruct hadronic top

- cut or fit methods 


\section{Re-discovery Methods}

- Simple methods first:

- "Cut and count" (C\&C): $\sigma_{\text {signal }}=\left(N_{\text {data }}-N_{\text {Background }}\right) /($ eff.xacc. $\times L)$

- Template fit to a discriminating variable $\rightarrow \sigma_{\text {signal }}$

- Cautious with event selection:

- Very simple/safe definition of measured objects.

- Missing $E_{T}$ poorly known at startup.

- Consider b-tag but use cautiously: Commissioning of b-tagging algorithms will take some time..

- Data-driven background measurements:

- Do not rely (too much) on simulation:

- Theoretical uncertainties (QCD-multijet, W+jets about 80-100\% ...)

- Acceptance uncertainties (lepton fake rate, b-tagging efficiency ...)

- Preferable to measure (main) backgrounds using data itself. 


\section{A note on b-tagging}

- b-tagging comissioning is ongoing:

- not envisaged in the first top re-discovery analyses presented here.

- as soon as the comissioning is completed it can be included: strongly suppresses the W+jets, QCD and Drell-Yan backgrounds.

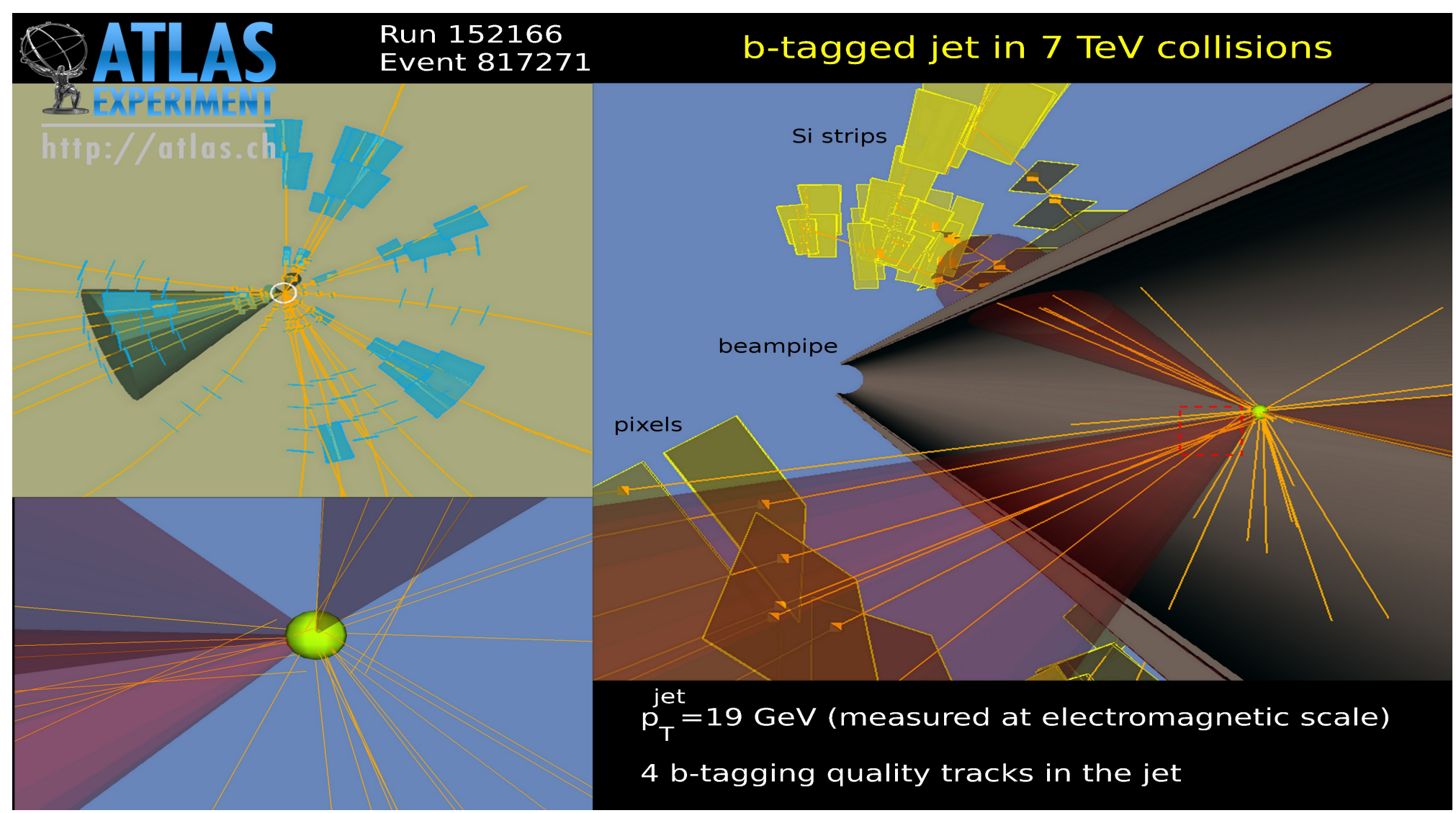




\section{ATLAS Lepton+jets @10 TeV}

- Basic event selection:

- high pT isolated lepton

- 4 high pT jets, 3 of them $>40 \mathrm{GeV}$

- In addition:

- Missing $E_{T}>20 G e V$

- Combine 3 jets to form hadronic Top (selection that maximizes $\mathrm{p}_{\mathrm{T}}$ top)

- 2 jets satisfy $W$ mass constraint ( $m W \pm 10 \mathrm{GeV}) \varepsilon=10 \%$.

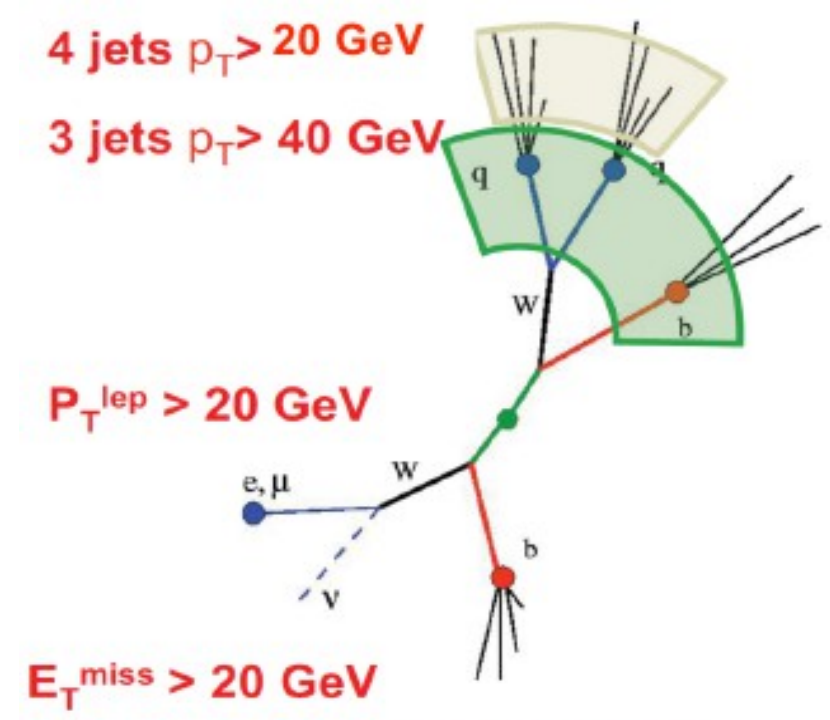

No b-tagging

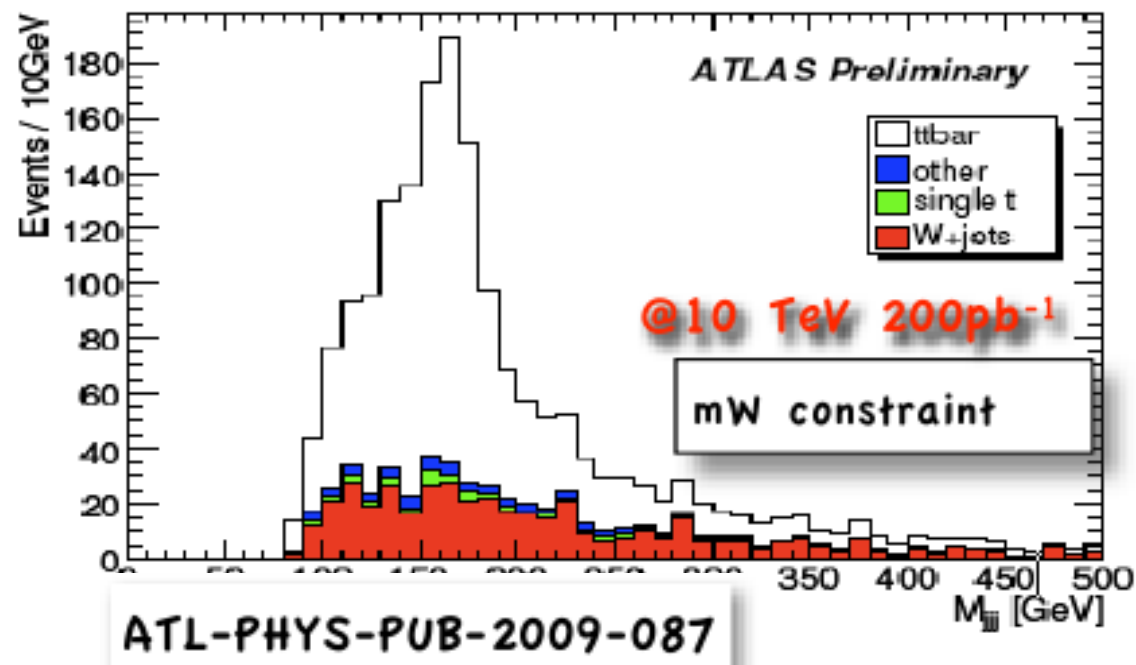

\begin{tabular}{|l|l|l|l|}
\hline $\begin{array}{l}200 \mathrm{pb}-1 \\
10 \mathrm{TeV}\end{array}$ & $\begin{array}{l}\text { Signal: el. } \\
\text { channel }\end{array}$ & backgrounds & S/B \\
\hline $\begin{array}{l}\text { After } \\
\text { selection }\end{array}$ & 1286 & 598 & 2.1 \\
\hline
\end{tabular}




\section{Atlas W+jets Data Driven Estimate}

- Event selection:

- require 2 leptons $80<m_{\|}<100 \mathrm{GeV}$ for $Z+$ jets and 1 lepton +MET for $W+j e t s$

- Use extrapolation:

- low jet multiplicity region as control region (CR) for $Z+$ jets (W+jets) events:

- Extrapolate ratio in the top signal region (SR): 4 jets or more

- Use ratio $W / Z$ to extrapolate $W$ into signal region:

$$
\left(\mathrm{W}^{\mathrm{SR}} / \mathrm{W}^{\mathrm{CR}}\right)_{\text {data }}=\left(\mathrm{Z}^{\mathrm{SR}} / \mathrm{Z}^{\mathrm{CR}}\right)_{\text {data }} \cdot C_{\mathrm{MC}} \quad C_{\mathrm{MC}}=\frac{\left(\mathrm{W}^{\mathrm{SR}} / \mathrm{W}^{\mathrm{CR}}\right)_{\mathrm{MC}}}{\left(\mathrm{Z}^{\mathrm{SR}} / \mathrm{Z}^{\mathrm{CR}}\right)_{\mathrm{MC}}}
$$

ATL-PHYS-PUB-2009-087

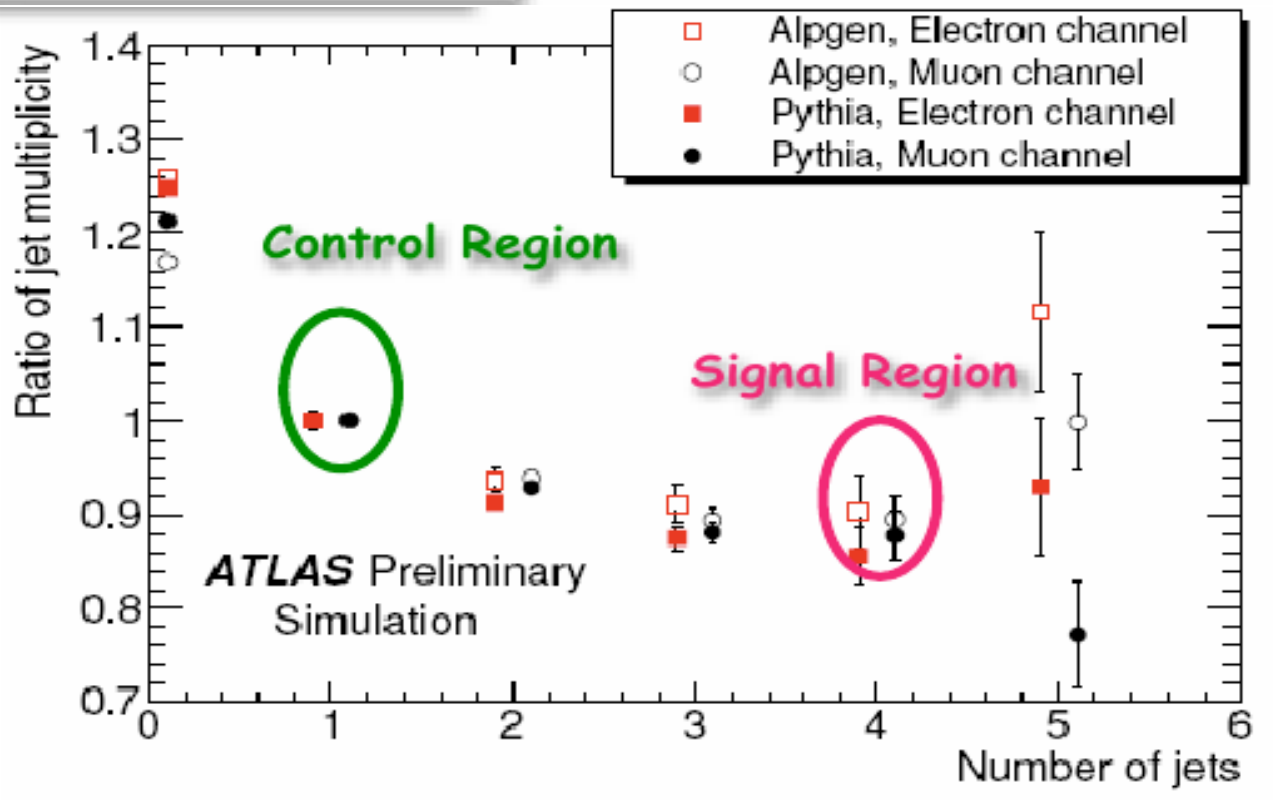

- Overall uncertainty:

- $20 \%$ @ $10 \mathrm{TeV}$ with $200 \mathrm{pb}^{-1}$ $50 \%$ @ 7 TeV with $10 \mathrm{pb}^{-1}$ $20 \%$ @ 7 TeV with 100 pb $^{-1}$

- Largest contributions:

- $50 \%$ uncertainty on QCD

- $C_{M C}: \sim 12 \%$ Pythia-Alpgen discrepancy 


\section{Two Main Methods to Extract cross-section}

- Cut and count method (C\&C):

- Advantage: simple method

- Disadvantage: Depends on background subtraction. Evaluate backgrounds from data!

- The fit or template fit to hadronic top mass:

- Advantage: much less sensitive to the Jet energy scale and background normalisation

- Disadvantage: relies on shape of distributions

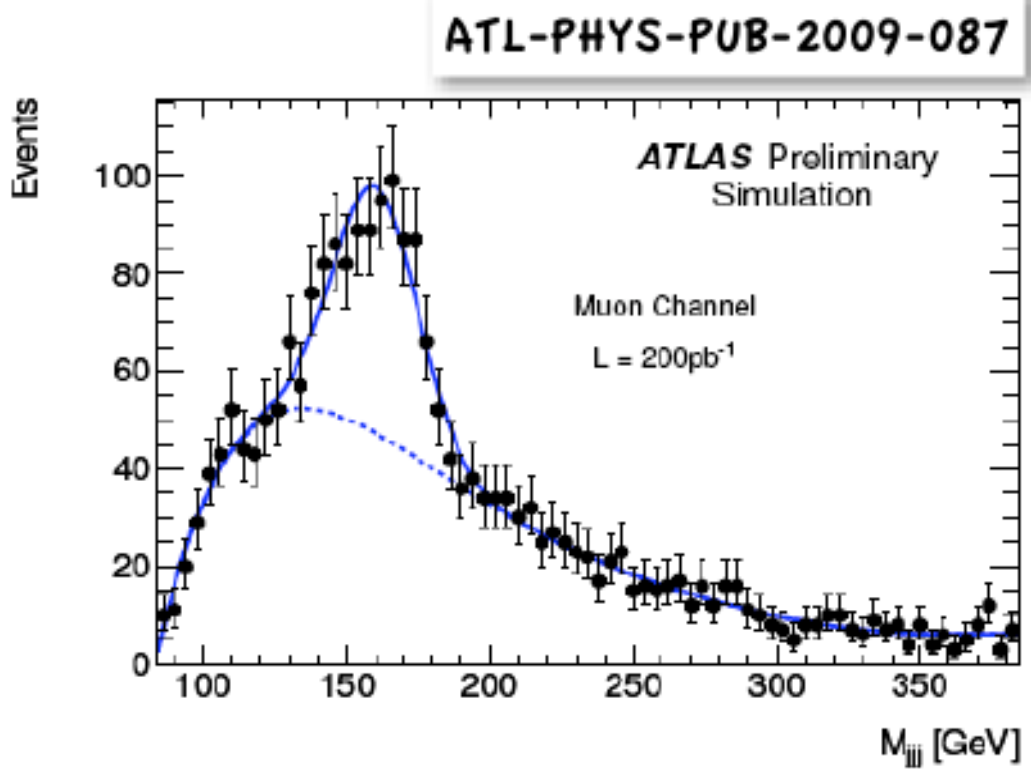

\section{$200 \mathrm{pb}^{-1} @ 10 \mathrm{TeV}$, electron channel}

\begin{tabular}{|l|c|l|}
\hline Method & \multicolumn{1}{|c|}{$\Delta \sigma / \sigma$} & Dominant systematics \\
\hline C\&C & $3.4(\text { stat })_{-21.1}^{+18.2}($ syst $) \pm 29.3$ (lumi) $\%$ & JES, ISR/FSR, W+jets \\
\hline Fit & $14(\text { stat })_{-15}^{+6}($ syst $) \pm 20($ lumi $) \%$ & JES, ISR/FSR \\
\hline No MET & $3.4(\text { stat })_{-25}^{+23}($ syst $) \pm 34($ lumi $) \%$ & JES, ISR/FSR, W+jets \\
\hline
\end{tabular}




\section{Top Re-discovery at $7 \mathrm{TeV}$ - lepton + jets}

- With 10 pb-1 notable signal:

- ATLAS will have 60 signal events with an expected background of 40 events per lepton flavor.

- Results before and after the additional $M_{w}$ cut are shown: 2 jets satisfying $W$ mass constraint ( $m W \pm 10 \mathrm{GeV}$ )

- Note that in addition b-tagging might be used:

S/B enhanced up to $\sim 5$ times!

Cross-section scaling:

$\sigma(t \bar{t})_{7} \cong 40 \% \sigma(t \bar{t})_{10}$

$\sigma(W+\text { jełs })_{7} \cong 45 \% \sigma(W+\text { jełs })_{10}$
Expected sensitivity for $10 \mathrm{pb}^{-1} @ 7 \mathrm{TeV}$

Scaled down from ATL-PHYS-PUB-2009-087 results

\begin{tabular}{|l|c|c|}
\hline Channel & el. analyis & $\mu$ analysis \\
\hline t† & 53 & 64 \\
\hline W+jets & 29 & 40 \\
\hline single top & 5 & 25 \\
\hline Other & 6 & 6 \\
\hline
\end{tabular}

Additional $M_{w}$ cut: $M_{j j}$ in $M_{w} \pm 10 \mathrm{GeV}$

\begin{tabular}{|l|c|c|}
\hline Channel & el. analyis & $\mu$ analysis \\
\hline t† & 26 & 32 \\
\hline W+jets & 10 & 14 \\
\hline single top & 2 & 2 \\
\hline Other & 2 & 2 \\
\hline
\end{tabular}




\section{ATLAS di-lepton Analysis}

\section{$200 \mathrm{pb}^{-1} @ 10 \mathrm{TeV}$}

- Basic event selection:

- 2 high pT (>20 GeV) isolated leptons

- $\geq 2$ jets $p T>20 \mathrm{GeV}$

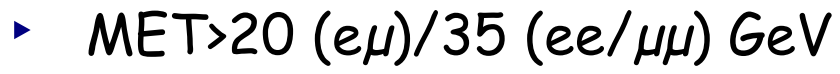

- veto $Z$ mass window

Cross-section estimation:

- Simple cut and count method

- Profile likelihood ratio

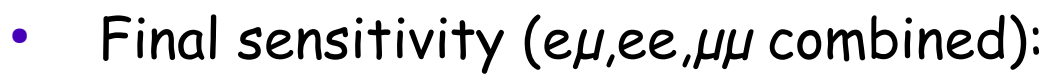

$$
\Delta \sigma / \sigma=\quad 3.1(\text { stat })_{-8.7}^{+9.6}(\text { syst })_{-17.4}^{+26.2}(\text { lumi }) \%
$$

Main syst: signal model, fake rate, Luminosity not included.
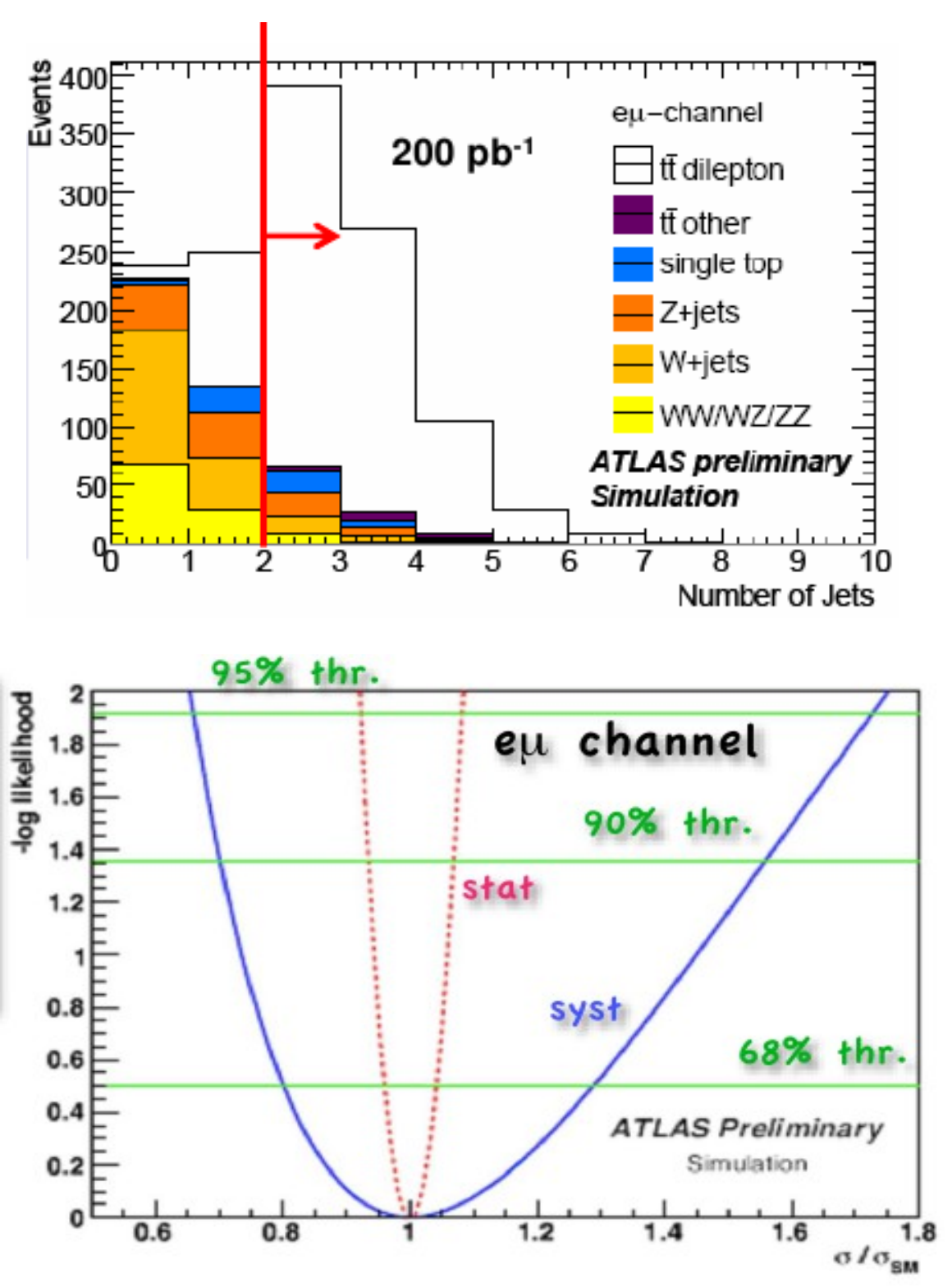


\section{Drell-Yan and Fake Leptons}

- Drell-Yan estimate from data: Drell-Yan mainly in G,H,I regions - top-antitop in A,C

- Use Z Monte-Carlo and data ( same for $C_{E s t}$ )

$E_{T}^{\text {MISS }}$

$$
A_{E s t}=G_{\text {Data }}\left(\frac{A_{M C}}{G_{M C}}\right)\left(\frac{B_{\text {Data }}}{H_{\text {Data }}}\right)\left(\frac{H_{M C}}{B_{M C}}\right)
$$

- Systematic uncertainty (stat. dependent!):

- Shift boundaries of the grid:

15\% for both channels @ $10 \mathrm{TeV}$ and $200 \mathrm{pb}^{-1}$

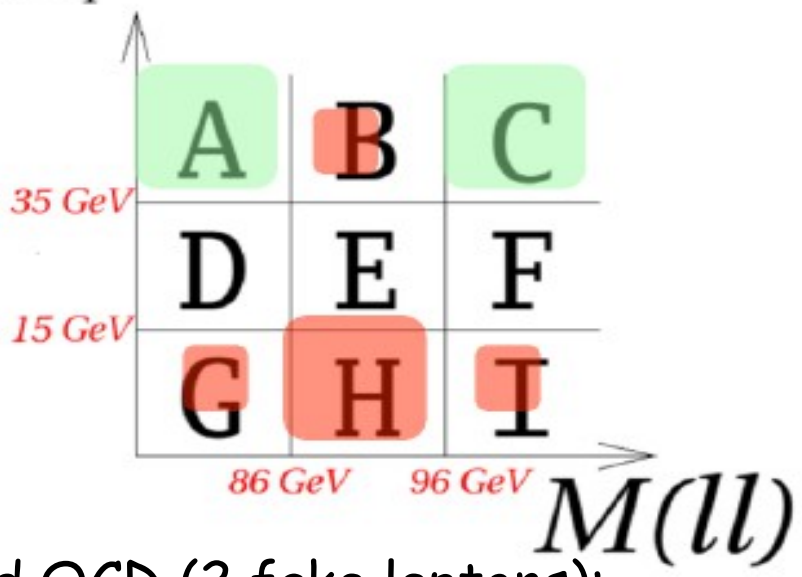

- Fake leptons in QCD jets: from W+jets (1 fake lepton) and QCD (2 fake leptons):

- muons:

- Decays in flight of $\pi$ or $K$ mesons in light jets

- Punch through muons

- Semileptonic decay in a heavy flavor $(b / c)$ jet

- electrons:

- Jets with high EM fraction

- Photon mis-associated with a track, conversions

Tracking Iso: $\sum \mathrm{p}_{\mathrm{T}}^{\text {track }}$ in $\eta-\phi$

Calorimeter Iso: $\sum \mathrm{E}_{\mathrm{T}}^{\text {tower }}$ in $\eta-\phi$

- Semileptonic decay in a heavy flavor (b/c) jet
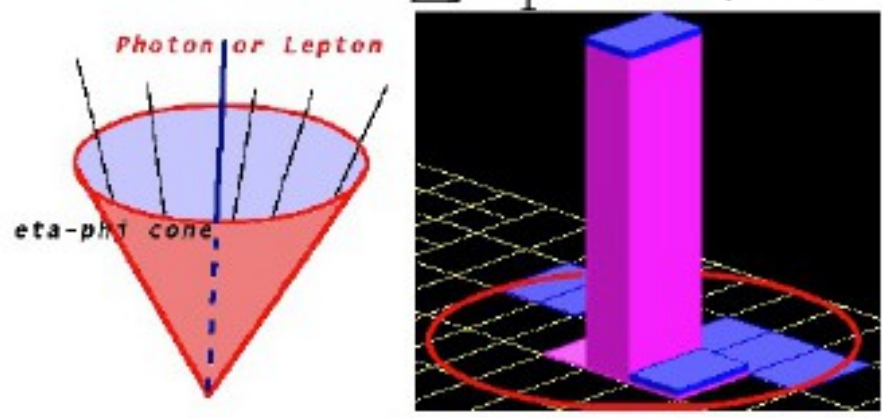


\section{Fake leptons in detail}

- Fake leptons from QCD jets: from W+jets (1 fake lepton) and QCD (2 fake leptons)

- Define a "tight( $T)$ " and a "loose $(L)$ " lepton, the latter enriched with fake leptons.

- Efficiencies to select a "tight $(T)$ " lepton for real $(R) \varepsilon$ and fake $(F) f$ leptons from data:

- $\varepsilon_{1 \mid 2}=N_{T, R} /\left(N_{T, R}+N_{L, R}\right)$ : Tag-and-probe on two leptons in $Z$ mass window, low MET required.

- $f_{1 \mid 2}=N_{T, F} /\left(N_{T, F}+N_{L, F}\right)$ : one (tight or loose) lepton and low MET required.

- $\varepsilon_{1 \mid 2}$ and $f_{1 \mid 2}$ for first|second lepton can exhibit $p_{T}$ or $n$ dependence.

- Neglecting the possibility of two fake leptons being reconstructed as tight leptons:

$$
\left[\begin{array}{c}
N_{\mathrm{TT}} \\
N_{\mathrm{TL}} \\
N_{\mathrm{LT}}
\end{array}\right]=\left[\begin{array}{ccc}
\epsilon_{1} \epsilon_{2} & \epsilon_{1} f_{2} & f_{1} \epsilon_{2} \\
\epsilon_{1}\left(1-\epsilon_{2}\right) & \epsilon_{1}\left(1-f_{2}\right) & f_{1}\left(1-\epsilon_{2}\right) \\
\left(1-\epsilon_{1}\right) \epsilon_{2} & \left(1-\epsilon_{1}\right) f_{2} & \left(1-f_{1}\right) \epsilon_{2}
\end{array}\right]\left[\begin{array}{c}
N_{\mathrm{RR}} \\
N_{\mathrm{RF}} \\
N_{\mathrm{FR}}
\end{array}\right]
$$

- Inverting and defining as fake all events with two tight leptons not coming from 2 real ones:

$$
N_{\text {Fake }}=\left[\frac{f_{2}\left(\epsilon_{2}-1\right)}{\epsilon_{2}-f_{2}}+\frac{f_{1}\left(\epsilon_{1}-1\right)}{\epsilon_{1}-f_{1}}\right] N_{\mathrm{TT}}+\frac{f_{2} \epsilon_{2}}{\epsilon_{2}-f_{2}} N_{\mathrm{TL}}+\frac{f_{1} \epsilon_{1}}{\epsilon_{1}-f_{1}} N_{\mathrm{LT}}
$$

- Rate uncertainty on MC 50-100\% (need data for estimation). 


\section{Top Re-discovery at $7 \mathrm{TeV}$ - dilepton}

- With $\sim 10 \mathrm{pb}-1$ convincing signal

- ATLAS will have 30 events with an expected background of 5 or 6 .

- Even with 5 pb-1, signal already plausible..

- At $1 \mathrm{pb}-1$, interesting event displays will start to appear at conferences

- Note that in addition b-tagging might be used.

\section{Expected sensitivity for $10 \mathrm{pb}^{-1}$ @7 TeV}

Scaled down from ATL-PHYS-PUB-2009-086 results

\begin{tabular}{|l|c|c|}
\hline Channel & $N($ signal $)$ & $N($ background $)$ \\
\hline$e-\mu$ & 14 & 2.5 \\
\hline$e-e$ & 4.3 & 1.1 \\
\hline$\mu-\mu$ & 6.6 & 1.9 \\
\hline Total & 25 & 5.5 \\
\hline
\end{tabular}

Cross-section scaling:

$$
\sigma(t \bar{t})_{7} \cong 40 \% \sigma(t \bar{t})_{10} \quad \sigma(W+\text { jets })_{7} \cong 45 \% \sigma(W+\text { jets })_{10}
$$




\section{Conclusions}

- Signatures of top pair signal will come fast

- Significant signal for "rediscovery" will require O(10pb-1)

- By the end of 2010 LHC might have statistics comparable to TeVatron.

- By the end of 2011 the samples will be significantly larger.

- Precision Standard Model physics: top quark properties.

- Hints of new Physics?

- Still a lot of work ahead:

- Trigger and lepton ID

- b-tagging

- Light jet and $b$ jet energy scale

- Missing $E_{T}$

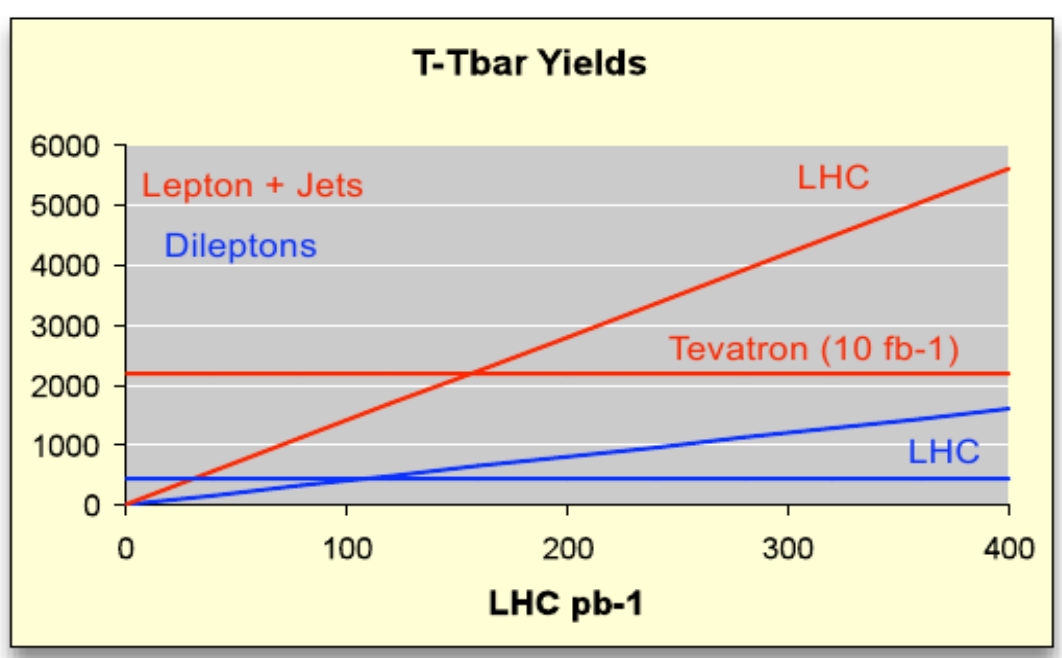

- Estimate backgrounds from data: $Q C D, W / Z+$ jets.. 